
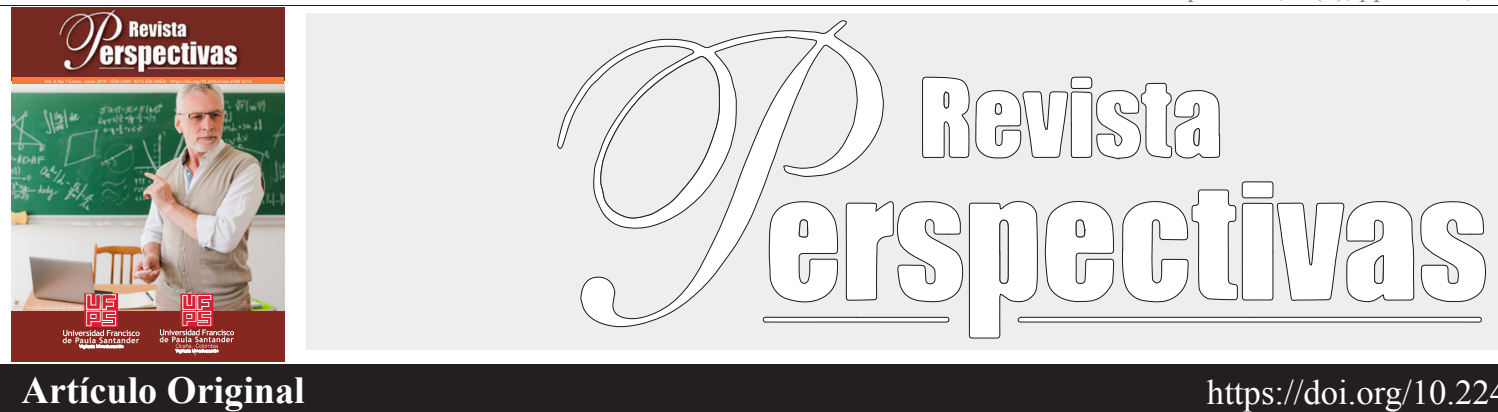

Artículo Original

https://doi.org/10.22463/25909215.1763

\title{
La construcción de ciudadanía en Colombia un hecho marcado por la violencia
}

The construction of citizenship in Colombia a fact marked by violence

A construção da cidadania na Colômbia é marcada pela violência

Sonia Verjel-Sánchez ${ }^{{ }^{*}}$

$a^{*}$ Magister en Gerencia de Mercadeo y Ventas, coordinación_ocana@fesc.edu.co, ORCID 0000-0002-8408-869X, Fundación de Estudios Superiores FESC, Ocaña, Colombia.

Cómo citar: Verjel, S. (2019). La construcción de ciudadanía en Colombia un hecho marcado por la violencia. Perspectivas, 4(1), 60-65.

Recibido: Agosto 20, 2018; Aceptado: Noviembre 01, 2018

\begin{tabular}{|c|c|}
\hline & RESUMEN \\
\hline Palabras Clave: & $\begin{array}{l}\text { El siguiente análisis plantea como la construcción de ciudadanía en Colombia, ha sido permeado directamente por la } \\
\text { violencia. El concepto de Ciudadanía moderna se relaciona a Thomas Humphrey Marshall, este sociólogo británico aboga }\end{array}$ \\
\hline & una posesión de derechos, lo haga también en términos de la incorporación \\
\hline & iedad de iguales. Para Marshall, la ciudadanía se va configurando a través de un \\
\hline Formación por competencias & uencial que parte con el desarrollo de la ciudadanía civil, como una condición necesaria aunque no suficiente \\
\hline Praxis del profesor & $\begin{array}{l}\text { de un posterior desarrollo de la ciudadanía política. Las constituciones políticas que adoptó Colombia, en su transcurrir } \\
\text { como nación hasta la actualidad, se ha tejido en torno al eje de las guerras y la violencia, sucesos que confirman el hecho } \\
\text { de que en Colombia se adquirieron primero los derechos políticos antes que los civiles (O'Donnell, 2001). El análisis } \\
\text { del estudio sobre Informe sobre la calidad de la ciudadanía y la democracia en Colombia } 2018 \text { (Pontificia Universidad } \\
\text { Javeriana, 2018), deja entrever como la ciudadanía en Colombia se ha construido sobre una débil institucionalidad, con la } \\
\text { presencia de un prolongado conflicto armado especialmente en las zonas rurales y la asociación de la política con prácticas } \\
\text { vinculadas a la corrupción, el clientelismo y el narcotráfico, denotan los escasos vínculos de confianza de los ciudadanos } \\
\text { entre sí y con las autoridades. }\end{array}$ \\
\hline
\end{tabular}

\section{ABSTRACT}

\section{Keywords:}

Meaningful learning Authentic evaluation Transversal competence Lecturer practice

The following analysis raises how the construction of citizenship in Colombia has been permeated directly by violence. The concept of modern citizenship is related to Thomas Humphrey Marshall, this British sociologist pleads for a citizenship that in addition to deciphering in a possession of rights, does so also in terms of the incorporation of the subject as a full member of a society of equals. For Marshall, the citizenship is configured through a sequential development that starts with the development of civil citizenship, as a necessary but not sufficient condition for the subsequent development of political citizenship. The political constitutions adopted by Colombia, in its passage as a nation until now, have been woven around the axis of wars and violence, events that confirm the fact that in Colombia political rights were first acquired before civil rights ( O'Donnell, 2001). The analysis of the study on the Report on the quality of citizenship and democracy in Colombia 2018 (Pontificia Universidad Javeriana, 2018), reveals how citizenship in Colombia has been built on a weak institutional framework, with the presence of a prolonged armed conflict especially in rural areas and the association of politics with practices linked to corruption, clientelism and drug trafficking, denote the limited bonds of trust between citizens and the authorities.

\section{RESUMO}

\section{Palavras chave:}

Aprendizagem significativa Avaliação autêntica Formação de habilidades Prática de professores
A análise a seguir propõe como a construção da cidadania na Colômbia tem sido diretamente permeada pela violência. O conceito de cidadania moderna está relacionado a Thomas Humphrey Marshall, esse sociólogo britânico defende uma cidadania que, além de ser decifrada na posse de direitos, também o faz em termos da incorporação do sujeito como membro pleno de uma sociedade de iguais. Para Marshall, a cidadania se configura através de um desenvolvimento seqüencial que começa com o desenvolvimento da cidadania civil, como condição necessária, mas não suficiente, para um posterior desenvolvimento da cidadania política. As constituições políticas adotadas pela Colômbia, em sua passagem como nação até agora, foram tecidas em torno do eixo das guerras e da violência, acontecimentos que confirmam o fato de que na Colômbia os direitos políticos foram adquiridos primeiro antes dos direitos civis (O'Donnell, 2001). A análise do estudo sobre o Relatório sobre a Qualidade da Cidadania e Democracia na Colômbia 2018 (Pontificia Universidad Javeriana, 2018) sugere que a cidadania na Colômbia foi construída sobre um quadro institucional fraco, com a presença de um conflito armado prolongado, especialmente em áreas rurais, e a associação da política com práticas ligadas à corrupção, clientelismo e tráfico de drogas. 


\section{Introducción}

El concepto de ciudadanía moderna se relaciona a Thomas Marshall, Citizenship and social Class (Marshall \& Bottomore, 1998), Marshall liga la ciudadanía moderna fundada sobre los derechos, al desarrollo de relaciones capitalistas y de la división del trabajo en el contexto del moderno Estado nacional.

La ciudadanía es un status que se adquiere al delimitar quiénes son los miembros de la comunidad que pueden reclamar ciertos derechos. Marshall aboga por una ciudadanía que además de descifrarse en una posesión de derechos, lo haga también en términos de la incorporación del sujeto como miembro pleno de una sociedad de iguales.

De acuerdo a, O’Donnell (2001), en América Latina la adquisición de los derechos del Ciudadano, presentó una secuencia diferente que la presentada en Europa, para O'Donnell, (2001), en Latinoamérica, se presenta una ciudadanía de baja intensidad donde derechos políticos coexisten con la negación de derechos civiles y sociales, y presenta el ejemplo de Argentina, donde los derechos sociales fueron primero que los derechos políticos y los derechos civiles se presentaron débiles o ausentes; a Colombia junto con Venezuela les ubica en la categoría de países que adquirieron primero los derechos políticos y donde existe una debilidad de los derechos civiles y sociales.

La historia de Colombia parece confirmar esta hipótesis y de igual manera se puede postular que en el país aún no se ha logrado consolidar un sentido común de ciudadanía tal como lo visualizó Marshall.

Relatan los libros de Historia, (Rodriguez Jimenez, 2010), que el 16 de marzo de 1781, en una plaza de mercado del Municipio de Socorro, Departamento de Santander, se iniciaron una serie de acontecimientos que darían inicio al proceso de independentista del país, un acto desafiante de una humilde mujer llamada Manuela Beltrán, frente a las autoridades de la Corona española, fijó el punto de partida hacia la independencia de Colombia de los Reyes de España.

Esta actitud de rebeldía se amplió por todo el territorio de la Nueva Granada, desde Mérida en Venezuela hasta el sur del Territorio, Pasto y Popayán, creándose una conciencia en el pueblo contra el gobierno colonial y la oligarquía criolla. Una revolución que marcó el despertar de un pueblo en búsqueda de su nación. Un "anhelo de liberación social" que se manifiesta en el "repudio de las masas oprimidas a los capitanes de la clase dominante" (Aguilera Peña, 1985).

Treinta años después de la revuelta del Socorro, un suceso recordado por el préstamo de un Florero, simboliza el llamado grito de la independencia, (Rodriguez Jimenez, 2010), relata que en la noche del 20 de julio de 1810, después de que el pueblo Santafereño se revelara ante el virreinato español, exigiendo el reconocimiento de sus derechos, se pactaron unos compromisos que definían la responsabilidad compartida del gobierno de la Nueva Granada. Los voceros del estamento criollo a espaldas del pueblo, establecieron una alianza para compartir el poder con quien representaba a la Corona española, reconociendo la autoridad del monarca Fernando VII y el Consejo de Regencia de España.

La cronología de los acontecimientos que han precedido el devenir histórico del país, se han escrito en una lucha constante de clases, de aquellas que a cualquier precio procuran mantenerse en el poder y de aquellos que buscan dignidad en su diario vivir.

Las constituciones promulgadas posteriores al hecho independentista definían al ciudadano de la época, sus deberes y responsabilidades para la nación que se formaba. De acuerdo a MacFarlene, (1994), una manera de entender el orden político que se conformó en la nueva Granada posterior al suceso emancipador, es el hecho de que se veían las constituciones simplemente como un instrumento de intereses de clases, que declaraban e instituían los derechos del hombre y el consenso de todo el pueblo y que escondían la determinación de las oligarquías criollas de promover sus beneficios socioeconómicos y evitar la difusión del poder entre el pueblo, sobre el que se basaba teóricamente la soberanía del estado.

De igual forma, MacFarlene, (1994), pone de manifiesto que otro enfoque para ver las constituciones iniciales de la naciente república, es el hecho que sus autores mostraron un genio notable para adoptar el lenguaje, estilo y entusiasmo de la época, mientras mantenían su propia herencia no democrática intacta.

La idea de ciudadanía que se estableció en la época post independista, declaró el rompimiento del vínculo social con la monarquía española y la restitución al pueblo en quien recaía la soberanía.

En el análisis realizado por Uribe de Hincapie, (1995), se configura los criterios o condiciones para acceder a la ciudadanía, consagrados en las sucesivas constituciones que desde 1810 a 1886 adoptó el país, en el proceso de conformación de la República; para la investigadora los conceptos de ciudadanía eran poco específicos y en muchos casos subjetivos, sometidos a un grado amplio de discrecionalidad. Condiciones como la ilustración (saber leer y escribir), la independencia económica (poseer determinada renta, pagar impuestos), el estado civil (casados), la etnia (esclavos, indígenas) y cualidades tales como el honor, la dignidad personal, la honradez, la moral. Como lo califica, Sosa Abella, (2009) ciudadano-propietario y un ciudadano ilustrado.

Rojas, (2008), divide la ciudadanía en Colombia del siglo XIX en tres periodos, el ciudadano que precedió los primeros cuarenta años de la independencia lo califica como el Ciudadano Patriota, por estar influenciados por sentimientos nacionalistas frente al poder opresor español. Hacia la mitad del siglo XIX predomina la imagen que la investigadora denomina ciudadano civilizado influenciado por un liberalismo ilustrado e individualizado europeo. En este periodo hace en- 
trada también la imagen del ciudadano soldado y soberano, las autoridades de la época proponían a Colombia como una nación en la que el ciudadano se convirtiera en soldado.

La visión de que la educación sería la alternativa más viable para formar el ciudadano requerido por el proyecto de nación, se convirtió en una de las principales preocupaciones para los sectores de poder y los grupos dirigentes, de acuerdo a los historiadores Quiceno, (2003), Laguna Rodriguez, (2014), la instrucción pública se constituyó como elemento uniforme y normalizador de toda la sociedad, crear la idea de comunidad, pasando por la de ciudadanía, patria y nación.

La instrucción pública impartida desde la Independencia tenía como objetivo generar identidad en los individuos, frente a unas instituciones y un Estado soberano, permeando a una población limitada de personas con la educación, por lo tanto se puede hablar de formación de identidad desde la alfabetización.

Dentro de las concepciones que adopta la idea de ciudadanía y ciudadano se destaca la promulgada en la Constitución de Rio Negro en 1863, como lo destaca, Otero Cleves, (2016), una constitución federativa, que al igual que las anteriores, reconoció los derechos individuales de los habitantes de la nación, pero en virtud del principio federal, dejó en manos de los estados soberanos la facultad de definir la ciudadanía política.

Los liberales se dieron a la tarea de educar un modelo particular de ciudadano. Por medio de la instrucción pública primaria y secundaria, de las escuelas normales, de las bibliotecas circulantes e incluso de la publicación de manuales del ciudadano, buscaron formar individuos conocedores de los derechos y deberes frente a la sociedad, y comprometidos a respetar un gobierno representativo y democrático.

Plantea (Uribe de Hincapié, 1998), que a lo largo del siglo XIX, las disputas entre republicanos y liberales radicales ocasionaron diferentes posturas predominantes con respecto a lo que debía ser un ciudadano, en el país se fueron configurando ciudadanías a las que llamo mestizas, a través de las cuales se ha desarrollado la lucha por los derechos, el reconocimiento y la inclusión.

Las constituciones políticas que adoptó Colombia, en su transcurrir como nación hasta la actualidad, se ha tejido en torno al eje de las guerras y la violencia.

De acuerdo a, Uribe de Hincapie M. , (1996), el país ha sido testigo de una sucesión de hechos violentos con diversas explicaciones para justificarlos y explicarlos, las guerras fueron asunto de pocos, de pequeños grupos que se reunían en torno a un partido, que participaban activamente en la política, que representaban a la nación en los cuerpos, colegiados y deliberaban en el Congreso, la guerra fue asunto de los ciudadanos, de los pocos ciudadanos realmente existentes, con clara conciencia de sus derechos, ilustrados sobre el significado del orden político y activos en la definición ideológica de sus colectividades, el motivo de las polémicas era muy diverso pero tenía un referente concreto: la constitución y la ley, para el colectivo común que era la mayoría de la población, poco tenían que ver con estas decisiones que terminaban envolviéndolos en el conflicto de diferente manera.

Como lo anota (O'Donnell, 2001), en Colombia se adquirieron primero los derechos políticos, antes que los civiles, en 1812, eran ciudadanos sufragantes los varones que cumplieran con los requisitos de ser: libres, mayores de 25 años o padres de familia, que vivían de sus rentas u ocupación sin dependencia de otra persona. Para 1832, la Constitución Política del Estado de Nueva Granada designó ciudadano a todo hombre casado o mayor de 21 años, que supiera leer y escribir y que tuviera la subsistencia asegurada.

En la Constitución de 1886, que perduró más de 100 años, se consideraban ciudadanos "los colombianos varones mayores de veintiún años que ejerzan profesión, arte u oficio, o tengan ocupación lícita u otro medio legítimo y conocido de subsistencia".

Lo anterior implicaba que las mujeres y las personas pobres o iletradas no tenían la condición de ciudadanos ni podían acceder a los derechos que la ciudadanía suponía (Giraldo García, 2015)

En el análisis realizado por (Otero Cleves, 2016). La Constitución de 1886, que precedió a la vigente en Colombia, impondría un ideal de ciudadanía más limitado y sustentado ya no en la libertad, sino en la idea del orden como base del progreso, restringió nuevamente el sufragio solo para aquellos que supieran leer y escribir o tuvieran una renta anual o propiedad determinada (art. 173), e invitó a una concepción de la ciudadanía basada en las ideas de orden público y la moral cristiana, por encima de la libertad individual.

La historia de la sociedad colombiana se ha caracterizado entre otros múltiples factores por la proliferación de la violencia y los conflictos, ha sido una violencia asociada a múltiples problemas sociales y políticos irresueltos, guerras que han enlutado a la mayor parte del territorio nacional y que han dejado miles de colombianos muertos e inmersos en el dolor.

Solo en el lapso de 1958 a 2012, el conflicto armado cobró la vida de 220.000 colombianos y de estas, el $80 \%$ han sido civiles, se trata de cientos de miles de víctimas fatales producto en especial de masacres, y asesinatos selectivos, a las que hay que sumar, muchos otros miles de víctimas de desaparición forzada, desplazamiento forzoso, secuestros, ejecuciones extrajudiciales, reclutamiento ilícito, tortura y sevicia, minas antipersonal, y violencia sexual (Centro Nacional de Memoria Histórica, 2013)

El ejercicio de la ciudadanía en Colombia, ha sido duramente impactado por la guerra, que no ha permitido el establecimiento de un orden republicano ciudadano, y es a su vez, esta falta de orden uno de los factores que han estimulado y posibilitado la permanente violencia a lo largo de la historia nacional.

Según (Pécaut, 1997), este hecho tiene que ver directamente con la precariedad en la construcción del Estado nación, es decir, la existencia de un cuerpo social dividido y fragmentado, un Estado sin autoridad y una no unificación simbólica de 
la nación. Bajo este contexto, lo que parece haberse dado en el país es una especie de coexistencia de ciudadanías parceladas que, a lo largo de la historia republicana, han entrado en constante conflicto y competencia.

(Valencia, 2017), refiere que Colombia es un país donde los partidos políticos son fuertes y tiene una de las democracias electorales más estables de la región; al mismo tiempo es un país con débiles reformas sociales y una larga historia de conflicto y de violación de derechos civiles, un país donde se combinan al mismo tiempo el orden y la violencia la estabilidad y el conflicto.

En este orden, Muñoz Monsalve, (2012), describe que en Colombia como en la mayoría de los países latinoamericanos la construcción de la nación y de la ciudadanía fue un proceso tan complejo como la conformación misma del estado nacional, porque nominalmente la ciudadanía debía incluir a toda la población pero en la práctica, operó como una categoría excluyente, implementada solo durante breves períodos y restringida para la mayoría de los habitantes, por razones diversas,

Para, (Uribe de Hincapié, 1998), la configuración del ciudadano y de sus derechos en Colombia ha sido resultado de un proceso histórico cultural en la constitución de estas ciudadanías ha predominado la tradición filosófica republicana sobre la liberal, los derechos colectivos sobre los individuales y las demandas del ejercicio del poder sobre los ordenamientos legales.

\section{La Nacionalidad y la Ciudadanía en la Constitu- ción Política de 1991}

Con el inicio de la década de los noventa el gobierno de Cesar Gaviria, anunció reformas y convocó a una Asamblea Nacional Constituyente, de acuerdo a (Hernández, 2016), la Constitución de 1991 nació como respuesta institucional a las varias formas de violencia que asolaban al país, uno de los motivos primordiales de la Asamblea Constituyente fue la necesidad de buscar la paz y restablecer el orden público, gravemente perturbado por las acciones del narcotráfico y de las organizaciones subversivas.

Para (Pécaut, 1997), la violencia se ha convertido en un modo de funcionamiento de la sociedad colombiana dando nacimiento a redes diversas de influencia sobre la población. A finales de los 80, a la ya larga lista de flagelos causantes de violencia en el país, se adicionó la del narcotráfico. Colombia vivió una década de violencia terrorista que derivó en la necesidad de realizar reformas estructurales sobre el funcionamiento del estado.

Con una sociedad dividida y fragmentada, con un Estado sin autoridad, la unidad simbólica de la Nación apenas si tenía la oportunidad de ser reconocida. El pluralismo de los partidos y de sus facciones haciendo las veces de democracia, no bastaba para suscitar un sentido de una ciudadanía común y menos todavía el de un espacio común de arreglo de los conflictos. (Pécaut, 1997).p.17

El 4 de Julio de 1991, el país adopta una nueva constitución, que da por terminada la de 1886 , la nueva carta política reconoció de manera explícita, el respeto a la dignidad de la persona, y el reconocimiento por parte del Estado de valores insustituibles como la libertad, la igualdad, la paz, el trabajo y la familia; se trató de dejar en claro que Colombia, es un Estado social y democrático de derecho, participativo, pluralista, y que uno de los objetivos principales de la organización estatal es garantizar la efectividad de los derechos, libertades, garantías y deberes de sus ciudadanos. (Hernández, 2016)

La nueva Constitución cambio la idea de Democracia representativa por la de Democracia participativa. Esto significa que los ciudadanos tendrán la posibilidad de participar más y de manera más directa en la toma de elecciones, contrario a la democracia representativa, en la que sólo se elegía a alguien para legislar, sin que el votante tuviera mayor participación en el poder político.

En la Constitución del 91 también se reconoció la diversidad étnica, cultural y religiosa de Colombia, lo que permitió que se aceptaran cosmovisiones y maneras de pensar diferentes.

Para, Garcia Sanchez, (2000), este cambio constitucional, que puede considerarse como el intento más grande que se ha dado en el siglo XX por establecer de una vez y por todas un pacto de paz, un orden republicano ciudadano y una nación cohesionada, estableció una ciudadanía multicultural, que supone que el Estado colombiano además de reconocer los derechos universales de que gozan los ciudadanos, lo hace también con los derechos colectivos de las comunidades negras e indígenas.

Retomando el planteamiento inicial, Marshall postula un desarrollo secuencial en la configuración de la ciudadanía, partiendo en una primera etapa con el desarrollo de la ciudadanía civil, para continuar luego con la ciudadanía política y culminar contemporáneamente con la ciudadanía social.

Esta concepción implica pensar el desarrollo de la ciudadanía civil como una condición necesaria aunque no suficiente de un posterior desarrollo de la ciudadanía política. Asimismo, supone que la ciudadanía política es una condición necesaria aunque no suficiente para el desarrollo de la ciudadanía social.

Para (Arias Murillo, 2006) América Latina ha vivido la ciudadanía en el marco de una realidad social, política y económica, donde el interés privado ha prevalecido sobre el interés del colectivo, En Colombia, por ejemplo, el artículo 58, de la carta magna garantiza la propiedad privada y los demás derechos adquiridos con arreglo a las leyes civiles, esto funda y afirma la propiedad como principio regulador de la ciudadanía; la propiedad determina las posibilidades de participación real en las decisiones y ejercicio gubernamental, lo cual conlleva la exclusión eficiente del grueso de la población. 


\section{Los significados de Ciudadanía para el Colom- biano}

A partir de la Constitución de 1991, El ejercicio de la ciudadanía en Colombia se entiende, como el posicionamiento consciente del individuo dentro de la estructura social y de su presencia al interior de la misma, que exige una participación activa y responsable en pro del mejoramiento de las condiciones de la vida de su comunidad.

En ese orden el individuo como integrante de la sociedad civil participa activamente en las acciones del Estado, la Constitución Nacional establece como un derecho del ciudadano la participación en la toma de decisiones, planeación, gestión, ejecución y control de la actividad pública. Lo anterior supone la participación activa de la población en los asuntos que se consideran monopolio del Estado.

Los regímenes democráticos suponen la existencia de mecanismos de participación ciudadana en el ejercicio del poder y en los asuntos públicos; adicional a esto, la gobernabilidad de un sistema político juega también un papel relevante, en tanto que está condicionada por la capacidad de los gobiernos para satisfacer las demandas de la población y mantener la estabilidad y el consenso político, lo cual sólo puede ser logrado si existen altos niveles de participación ciudadana activa (Pérez, 1994).

La Participación Ciudadana tiene que ver con vivir la democracia. Es ejercer el derecho a elegir y ser elegido, el derecho a opinar, a participar en plebiscitos, referendos y consultas populares, constituir partidos y movimientos políticos, la posibilidad de revocatoria del mandato, la iniciativa legislativa y el cabildo abierto, etc.

La participación la ejercen todas las personas sin distingo de sexo, raza o condición. Se extiende también a las empresas como ciudadanos y la sociedad civil.

La Constitución Política Nacional de Colombia de 1991 describe en los Artículos 40 y 103 lo relacionado con la participación y el control social, señalando además, cuáles son las formas y los mecanismos de esa participación y control.

Para, (Peralta, 2010), el ejercicio de la ciudadanía en Colombia ha sido un ejercicio limitado por la rehegemonización del mercado y la colonización, por parte de éste, del principio del Estado y del principio de la comunidad, la difusión social del trabajo y el aislamiento político del mismo, no sólo han inhibido el desarrollo de un tipo de ciudadanía cuyos límites o factores determinantes podrían ubicarse por fuera de la teoría liberal, sino que además se ha propiciado un tipo de ciudadanía en la cual la participación ha quedado reducida al ejercicio electoral y/o derecho al voto.

En el país, el ejercicio de la ciudadanía en todas sus dimensiones ha encontrado obstáculos en el orden republicano, lo que no ha permitido la incorporación del sujeto como miembro pleno de una sociedad de iguales como lo visualizó Marshall.

Un presente estudio realizado por (Pontificia Universidad
Javeriana, 2018), para la Registraduría Nacional de Colombia sobre la Calidad de la Ciudadanía, revela la crisis que se presenta en las instituciones de justicia y seguridad del país.

En este estudio fue aplicada una encuesta dirigida a 4.560 colombianos, mayores de edad, hombres y residentes en las ciudades principales y en municipios intermedios y pequeños de la geografía colombiana, en la cual se realizaron preguntas relacionadas al Estado de derecho y acceso a la justicia, vida comunitaria, redes de asociación, vida política (participación política), sociedad civil y participación no electoral, valores y calidad de la ciudadanía y redes sociales.

En el análisis efectuado por (Avila, 2019), sobre el mencionado informe revela que el $52 \%$ de los colombianos dicen que las leyes no se cumplen nada, un $7 \%$ dicen que se cumplen, mientras que un $41 \%$ manifestaron que se cumplen medianamente. En lo fundamental, la población percibe una corrupción alta.

Además, ante robos, homicidios o agresiones, las instituciones del Estado tardan en responder y la justicia por mano propia comienza a surgir como una posibilidad. Un $15 \%$ de los encuestados confirmaron la posibilidad de hacer justicia por mano propia y un $74 \%$ estarían dispuestos a retener algún criminal hasta que las autoridades llegaran.

Demoras en el proceso y falta de confianza en las instituciones son las principales barreras para acceder a las instituciones de justicia. Sobre el tema de la salud de la democracia colombiana, hay temas alentadores sobre la participación política, pero otros donde aún falta un largo camino por recorrer.

A todo lo anterior se le suma la percepción de la población sobre la transparencia en las elecciones. Los resultados muestran que el $46 \%$ de los encuestados manifiestan que no son nada trasparentes, el $40 \%$ medianamente transparentes y solo un $13 \%$ las consideran muy transparentes.

La protección de los ciudadanos y la limitación de los regímenes políticos fundamentos de una concepción liberal-democrática de ciudadanía por la cual se apeló en Colombia desde los inicios republicanos, y que se reafirmó en el proceso constituyente que se consolidó en el pacto político y social de 1991 y en los desarrollos legales y judiciales de la Constitución, se perciben claramente fragmentados.

La falta de seguridad de los ciudadanos, la arbitrariedad y el autoritarismo por parte de los gobernantes y de los demás funcionarios, las dificultades que se tienen para el ejercicio de la acción colectiva, la movilización social, la defensa de los derechos humanos y la que se ha denominado como criminalización o estigmatización de la protesta social en Colombia.

El ejercicio de la ciudadanía en Colombia, presenta avances y retrocesos con relación a el afianzamiento del Estado de derecho, de la legalidad como principio para la vida social y política en el país y del acceso a la administración de justicia y su eficacia frente a los ciudadanos cuando reclaman la vulneración de sus derechos; la inclusión de grupos discriminados; los desafíos que conlleva la vida comunitaria y el empoderamiento frente a problemáticas locales, así como el reconocimiento de 
actores colectivos en lo regional

La ciudadanía en Colombia se ha construido sobre una débil institucionalidad, la presencia de un prolongado conflicto armado especialmente en las zonas rurales, la asociación de la política con prácticas vinculadas a la corrupción, el clientelismo y el narcotráfico y los escasos vínculos de confianza de los ciudadanos entre sí y con las autoridades.

Este escenario ha llevado a la consideración de lo público como fuente de beneficios individuales, reduciendo el valor del trabajo colectivo y de los valores democráticos sobre los que se fundó el proyecto constitucional de 1991. (Pontificia Universidad Javeriana, 2018)

En la actualidad y 28 años después de adoptada la Constitución, Colombia no ha logrado la construcción de un proyecto político lo suficientemente sólido y amplio en el que se dé cabida a la mayoría de los miembros del territorio nacional, en el que todos los colombianos se sientan en unidad. El ejercicio de una ciudadanía plena se queda en retórica, toda vez que el reconocimiento en la práctica encuentra su dificultad y esa visión popular que los derechos son privilegios de pocos se ha arraigado en el colectivo social

\section{Referencias}

Aguilera Peña, M. (1985). Los Comuneros Guerra Social Y Lucha Anticolonial. Bogotá: Universidad Nacional de Colombia.

Arias Murillo, F. A. (2006). Ciudadanía en el contexto democrático de América Latina. Hallazgos, 151-165.

Avila, A. (05 de Marzo de 2019). La calidad de la ciudadanía y la democracia en Colombia. El País, pág. 10.

Giraldo García, A. (2015). El concepto de Ciudadania en Colombia: Evolución Historica y aportes socioculturales. Revista de Estudiantes de Ciencia Politica, 6, 58-71.

Hernández, J. G. (19 de 04 de 2016). La Constitución de 1991: 25 años de un proyecto humanista y democrático. Razón Pública. Obtenido de https://www.razonpublica.com

Marshall, T., \& Bottomore, T. (1998). Ensayo Ciudadania y Clase Social. Madrid: Alianza Editorial.

O'Donnell, G. (2001). "Reflections on Contemporary South American". Journal of Latin American Studies, 33, 599609.

Otero Cleves, A. M. (2016). Constituciones y ciudadanía en el siglo XIX colombiano. Bogotá: Subgerencia Cultural del Banco de la República - Bogotá / Biblioteca Luis Ángel Arango. Recuperado de: http://proyectos. banrepcultural.org/asamblea-nacional-constituyente/ es/texto-catalogo/constituciones-y-ciudadan $\% \mathrm{C} 3 \% \mathrm{~A}-$ Da-en-el-siglo-xix-colombiano.

Pécaut, D. (1997). "Presente pasado y futuro de la violencia". Análisis Politico, No.30. Instituto de Estudios Politicos y Relaciones Internacionales. Universidad Nacional de Colombia, 1-43.

Peralta, B. (2010). La forma en que se ejerce la ciudadania en la democracia Colombiana, socialización politica y cultura ciudadana. Eleuthera Vol. 4, enero - diciembre, 253-290.

Pérez, J. (1994). Descentralización Educativa. Revista Iberoamericana de Educación.

Pontificia Universidad Javeriana. (5 de Marzo de 2018). Informe sobre la calidad de la ciudadanía y la democracia en Colombia 2018. Bogotá: Ediciones Berea.

Rodriguez Jimenez, P. (2010). Historia que no Cesa, LA INDEPENDENCIA DE COLOMBIA, 1780-1830. Bogotá.: Universidad del Rosario.

Uribe de Hincapié, M. T. (1998). Órdenes complejos y ciudadanías mestizas. Una mirada al caso Colombiano. Estudios Poiticos, No. 12, 25-46.

Valencia, A. (2017). Daniel Pecaut. En busca de la nación colombiana: Conversaciones con Alberto Valencia Gutierrez. Bogotá: Grupo Editorial Colombia. 